\title{
Improving the efficiency of white LEDs
}

Shunsuke Murai, Gabriel Lozano, Marc A. Verschuuren, Said R. K. Rodriguez, and Jaime Gómez Rivas

Diffractive arrays of metallic nanoparticles act as nanoantenna phased arrays that enhance emission from thin phosphor layers in defined directions, and therefore offer new prospects for solid-state lighting.

The solid-state lighting revolution-the rapid replacement of incandescent lamps with more efficient light sources-has been made possible by the introduction of white LEDs. ${ }^{1}$ LEDs can be used to produce white light via two different approaches. In the first approach, several LEDs emit different color light, which is combined at the correct intensities to produce white light. This method is limited by the low quantum efficiency (QE) of green LEDs and the temperature dependence of red LEDs. Therefore the second approach, which uses high-efficiency UV or blue LEDs to excite luminescence of a high-QE material (a phosphor), is more widely used. Much effort is now being dedicated to the development of stable phosphors with the highest possible QE.

Secondary optics (i.e., lenses and mirrors) are important parts of LEDs that are used to couple the light emitted from the devices and to beam this light in defined directions. Intensive research is currently focused on single or few emitters, low-QE emitters, and/or IR emitters that are coupled to gold nanoantennas. However, nanoantennas with those characteristics have limited use for solid-state lighting applications, which require the redirection of sufficient light emission from high-QE emitters at visible wavelengths.

We have recently demonstrated new methods to improve the emission characteristics of phosphors (i.e., spectrum and direction), by combining them with arrays of metallic nanoparticles. The nanoparticles support localized surface plasmon resonances, which are the result of coherent oscillation of electrons in the structure. These optical resonances, together with the small nanoparticle dimensions (few tens to hundreds of nanometers), allow the arrays to act as antennas for light. ${ }^{2}$ We have used arrays of silver and aluminum nanoparticles, coupled to phosphors that have been developed for white LEDs, to make large (a)

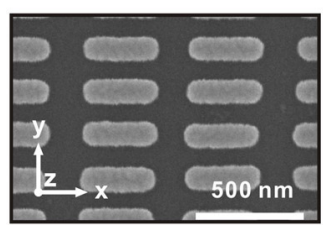

(b) Ag nanoparticle

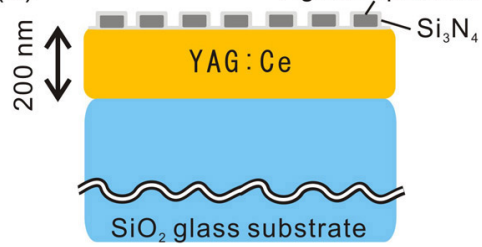

Figure 1. (a) Scanning electron microscope image of the top view of a silver (Ag) nanorod array. (b) A schematic side view of the sample. The nanoantenna array sits on top of a cerium-doped yttrium aluminum garnet (YAG:Ce) layer and was synthesized on a silica $\left(\mathrm{SiO}_{2}\right)$ glass substrate. $\mathrm{Si}_{3} \mathrm{~N}_{4}$ : Silicon nitride.

directional enhancements of the phosphors' emissions, as well as to make strong modifications of their absorption and emission spectra. ${ }^{3,4}$ These nanoparticle arrays share some similarities with antenna phased arrays, which beam electromagnetic waves in directions that are defined by the interference of radiation from elements in the array.

A scanning electron microscope image of a silver nanoparticle array is shown in Figure 1(a). This array was fabricated using substrate-conformal imprint lithography. ${ }^{5}$ The separation distance between the nanoparticles in the array is on the order of visible light wavelengths, thus making the array a diffraction grating. A schematic cross section of the phosphor and the antenna array is shown in Figure 1(b). The antenna array is on top of the light-emitting layer of cerium $\left(\mathrm{Ce}^{3+}\right)$-doped yttrium aluminum garnet (YAG:Ce). This important luminescent material was synthesized using the sol-gel method on a silica $\left(\mathrm{SiO}_{2}\right)$ glass substrate. ${ }^{6}$ This sample supports collective hybrid plasmonic-photonic modes that are known as surface lattice resonances. These result from the diffractive coupling of localized surface plasmons in the individual particles. The hybrid plasmonic-photonic modes are characterized by a field enhancement that extends over volumes larger than those associated with the localized surface plasmons of the individual nanoparticles. The sample can also support guided modes in the 
(a) Extinction

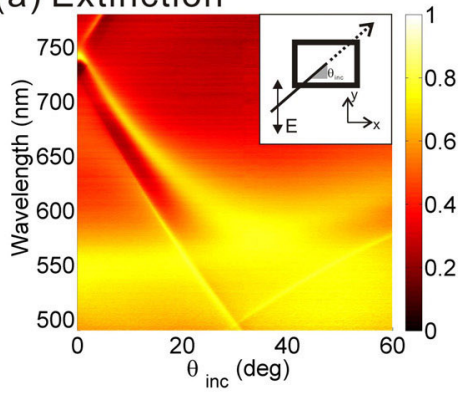

(b) Emission

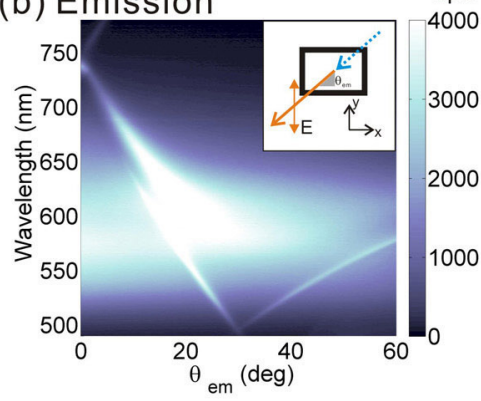

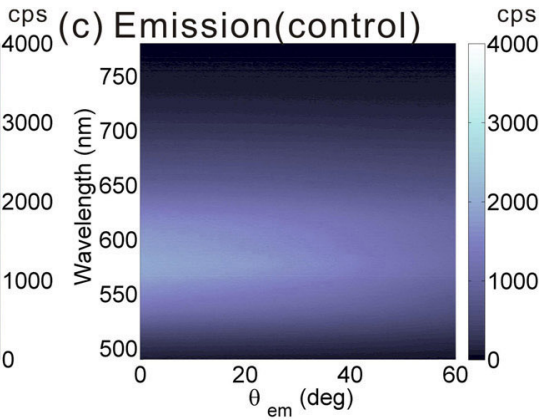

Figure 2. (a) Extinction (as the color scale) of a silver nanoparticle array on top of a YAG:Ce layer, as a function of the wavelength and the angle of incidence $\left(\theta_{i n c}\right)$. The incident light is polarized along the $y$-axis, i.e., parallel to the short axis of the nanorods. The extinction is defined as 1-T where $T$ is the $0^{\text {th }}$-order transmittance. The inset shows a schematic illustration of the experiment, where $E$ is the electric field. (b) Emission spectrum (polarized along the y-axis) of the YAG:Ce, as a function of the wavelength and the angle of emission, with respect to the normal to the surface $\left(\theta_{\text {em }}\right)$. The inset shows a schematic illustration of the experiment. (c) Emission spectrum for a control YAG:Ce layer without a nanorod array. deg: Degrees. cps: Counts per second.

YAG:Ce layer that can couple to free-space radiation via scattering from the nanoantenna array.

As shown in Figure 2(a), the extinction of the spectrum produced from our nanoparticle array varies with the light's angle of incidence. We observe a broad and angle-independent extinction band at about 540nm, which is due to the localized surface plasmons of the nanoparticles. We also see sharp and dispersive bands with high extinction. These bands correspond to the extinction of surface lattice resonances and the coupling of incident light to the fundamental guided mode of the YAG:Ce layer. We used a blue (442nm) laser to excite the YAG:Ce layer and measured the emission spectrum-see Figure 2(b) - as a function of the emission angle. Our measurements on a control sample without the array-see Figure 2(c)—display a broad yellow emission that is typical of YAG:Ce. The emission spectrum from our sample, however, is very different from that of the control. The emission intensity is greater and the spectral shape is significantly modified. The shape of the emission spectrum is similar to that of the extinction. This is because the excited YAG:Ce decays by emitting light into the modes of the array, which are coupled to free-space radiation in certain preferential directions that define the mode dispersion. The extinction and emission of light from our nanoparticle array are analogous to the receiving and radiating of electromagnetic waves by an antenna.

We have also investigated arrays of aluminum and gold nanoparticles, combined with quantum dots and polymer films that contain fluorescent organic dye molecules. An aluminum array with dye molecules, illuminated by a blue laser, is shown in Figure 3. ${ }^{7}$ The white light corresponds to the emission of the

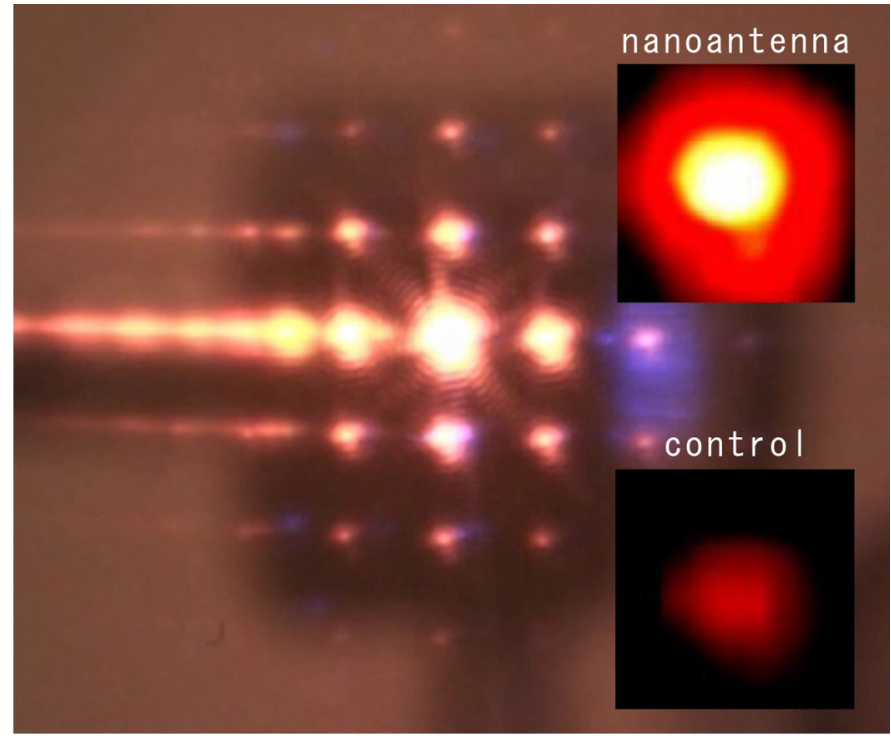

Figure 3. Photograph of a sample that consists of a periodic array of aluminum nanoparticles and an emitting layer containing dye molecules. A blue laser beam is incident from the backside and creates a bright spot on the sample. The multiple spots are due to the diffraction of the array. The insets compare the emission intensity at the irradiation spot. The sample with the nanoantenna array is much brighter than the control sample without the array.

dye, combined with the fraction of unabsorbed blue light. The multiple bright spots arise from the diffraction orders of the array that are trapped and guided in the glass substrate. 
The emission intensity of the dye-with a high QE of 0.85 - is enhanced up to 70 times in the forward direction by the nanoparticle array. The emission directionality also can be precisely controlled by the pitch of the array. ${ }^{8}$

We have developed new metallic nanoantenna arrays and demonstrated their performance. These samples can be used to enhance the emission of phosphors in defined directions and therefore improve the efficiency of white LEDs. To achieve even greater enhancement of phosphors, we now plan to further optimize the geometries of our antennas and the thickness of the phosphor layers.

\section{Author Information}

\section{Shunsuke Murai}

Kyoto University

Kyoto, Japan

Shunsuke Murai received his PhD in engineering in 2011 from Kyoto University, Japan. He has been working as an assistant professor since 2004. He has also been a Japan Science and Technology Agency Precursory Research for Embryonic Science and Technology (PRESTO) researcher since 2013.

Gabriel Lozano, Said R. K. Rodriguez, and Jaime Gómez Rivas FOM Institute for Atomic and Molecular Physics (AMOLF)

Eindhoven, The Netherlands

\section{Marc A. Verschuuren}

Philips Research Laboratories

Eindhoven, The Netherlands

\section{References}

1. S. Pimputkar, J. S. Speck, S. P. DenBaars, and S. Nakamura, Prospects for LED lighting, Nat. Photon. 3, pp. 180-182, 2009.

2. P. Bharadwaj, B. Deutsch, and L. Novotny, Optical antennas, Adv. Opt. Photon 1, pp. 438-483, 2009.

3. S. R. K. Rodriguez, S. Murai, M. A. Verschuuren, and J. Gómez Rivas, Light-emitting waveguide-plasmon polaritons, Phys. Rev. Lett. 109, p. 166803, 2012 doi:10.1103/PhysRevLett.109.166803

4. S. Murai, M. A. Verschuuren, G. Lozano, G. Pirruccio, S. R. K. Rodriguez, and J. Gómez Rivas, Hybrid plasmonic-photonic modes in diffractive arrays of nanoparticles coupled to light-emitting optical waveguides, Opt. Express 21, pp. 4250-4262, 2013.

5. M. A. Verschuuren and H. van Sprang, 3D photonic structures by sol-gel imprint lithography, Mater. Res. Soc. Proc. 1002, pp. N03-N05, 2007. doi:10.1557/PROC1002-N03-05

6. S. Murai, M. A. Verschuuren, G. Lozano, G. Pirruccio, A. F. Koenderink, and J. Gómez Rivas, Enhanced absorption and emission of $\mathrm{Y}_{3} \mathrm{Al}_{5} \mathrm{O}_{12}: \mathrm{Ce}^{3+}$ thin layers prepared by epoxide-catalyzed sol-gel method, Opt. Mater. Express 2, pp. 1111-1120, 2012 7. G. Lozano, D. J. Louwers, S. R. K. Rodriguez, S. Murai, O. T. A. Jansen, M. A. Verschuuren, and J. Gómez Rivas, Plasmonics for solid-state lighting: enhanced excitation and directional emission of highly efficient light sources, Light: Sci. Appl. 2, p. e66, 2013. doi:10.1038/lsa.2013.22

8. G. Lozano, G. Grzela, M. A. Verschuuren, M. Ramezani, and J. Gómez Rivas, Tailor-made directional emission in nanoimprinted plasmonic-based light-emitting devices, Nanoscale 6, pp. 9223-9229, 2014 